Benedetto Bongiorno and Luisa Di Piazza* Department of Mathematics, University of Palermo, Via Archirafi 34, 90123 Palermo, Italy. e-mail:

bb@dipmat.math.unipa.it and dipiazza@dipmat.math.unipa.it

Valentin Skvortsov ${ }^{\dagger}$ Department of Mathematics, Moscow State University, Moscow 119899, Russia and Instytut Matematyki, WSP, Bydgoszcz, 85-065

Poland. e-mail: skworcow@wsp. bydgoszcz.pl

\title{
ON THE $n$-DIMENSIONAL PERRON INTEGRAL DEFINED BY ORDINARY DERIVATES
}

\begin{abstract}
We introduce an $n$-dimensional Perron integral defined in terms of ordinary (in the Saks terminology) derivates. We prove that we get an equivalent definition of this integral if in the definition we use only continuous major and minor functions. We also prove that this integral is equivalent to a version of the Kurzweil-Henstock integral defined by Mawhin.
\end{abstract}

\section{Introduction}

It is well known (see [16]) that the classical Perron integral defined on an interval of the real line $\mathbb{R}$ by continuous major and minor functions is equivalent to the one defined by major and minor functions which are not assumed to be continuous. A proof of this fact based on the Kurzweil-Henstock theory of integration is given in [17] (see also [2]). In the multidimensional case this equivalence was established in [12] for the Perron integral defined with respect to the regular interval basis (with a fixed regularity) and in [1] for the case of the full interval basis (without any regularity condition).

\footnotetext{
Key Words: Perron integral, ordinary derivates, derivation basis, variation.

Mathematical Reviews subject classification: Primary: 26A39, 26A42, 26 A45.

Received by the editors October 21, 2000

* Supported by M.U.R.S.T. of Italy and by "Accordo quadro tra l'Università di Palermo e l'Università Lomonosov di Mosca".

†Supported by RFFI (99-01-00355) and by "Accordo quadro tra l'Università di Palermo e l'Università Lomonosov di Mosca".
} 
Here we proceed with this kind of investigation considering a Perron-type integral which we define using so called ordinary (in the Saks terminology, see [16], Ch. IV) upper and lower derivates. We shall call it the ordinary Perron integral or $P$-integral. This notation will lead to no confusion here, because in this paper we never use the Perron integral defined by strong derivates which is equivalent to the usual $n$-dimensional Kurzweil-Henstock integral and is often referred to as the $P$-integral. We prove in Theorem 1 that if we suppose that major and minor functions in the definition of the $P$-integral are continuous (in a suitably defined sense), then we get an integral ( $P^{c}$-integral) which is equivalent to the $P$-integral. Moreover we prove that both of these integrals are equivalent to the Mawhin integral of [11] (see also [7], [5] and [4]). The proof essentially depends on the continuity property of the $\delta$-variation generated by the indefinite integral. We also consider some other versions of the definition of the $P$-integral.

\section{Preliminaries}

We recall some definitions and notation. In this paper the word "measure" as well as the expression "almost everywhere" always refers to $n$-dimensional Lebesgue measure on $\mathbb{R}^{n}$, denoted by $|E|$ for a set $E \subset \mathbb{R}^{n}$. In $\mathbb{R}^{n}$ we use the norm $\|x\|=\max _{1 \leq i \leq n}\left|x_{i}\right|$, where $x=\left(x_{1}, x_{2}, \ldots, x_{n}\right)$. Then the $\delta$ neighborhood of $x$, denoted by $U(x, \delta)$, is an open cube centered at $x$ with sides equal to $2 \delta$. Int $E$ denotes the interior of $E$.

An interval in $\mathbb{R}^{n}$ is a set $I=\left[a_{1}, b_{1}\right] \times\left[a_{2}, b_{2}\right] \times \cdots \times\left[a_{n}, b_{n}\right]$ with $a_{i}<b_{i}$, $i=1,2, \ldots, n$. We call the number $r(I)=\min _{i}\left(b_{i}-a_{i}\right) / \max _{i}\left(b_{i}-a_{i}\right)$ the regularity of the interval $I$. Let $\alpha \in(0,1)$; if $r(I)>\alpha$ we say that the interval $I$ is $\alpha$-regular. Throughout this paper $\Delta$ denotes a fixed interval and $\mathcal{I}$ the family of all subintervals of $\Delta$.

We consider here two derivation bases on $\Delta$. By $\mathcal{F}$ we denote the so called full interval basis; i.e., the subset of $\mathcal{I} \times \Delta: \mathcal{F}=\{(I, x): I \in \mathcal{I}, x \in I\}$. We shall also use the regular subbases of $\mathcal{F}$ defined as follows: if $\alpha \in(0,1)$, by $\mathcal{F}^{\alpha}$ we denote the basis of all pairs $(I, x) \in \mathcal{F}$ with $r(I)>\alpha$ and we call it the $\alpha$-regular basis.

In the following definitions and notation $\mathcal{B}$ stands either for $\mathcal{F}$ or for $\mathcal{F}^{\alpha}$. For a set $E \subset \Delta$ we write

$$
\mathcal{B}(E)=\{(I, x) \in \mathcal{B}: I \subset E\} \text { and } \mathcal{B}[E]=\{(I, x) \in \mathcal{B}: x \in E\} .
$$

A positive function $\delta$ defined on a set $E \subset \Delta$ is called a gauge on $E$. A gauge on $\Delta$ is called simply a gauge. For a given gauge we set

$$
\mathcal{B}_{\delta}=\{(I, x) \in \mathcal{B}: I \subset U(x, \delta(x))\} .
$$


The meaning of $\mathcal{B}_{\delta}(E)$ and $\mathcal{B}_{\delta}[E]$ is clear from (1) and (2). It is clear that to define $\mathcal{B}_{\delta}[E]$ it is enough to have a gauge defined only on $E$. A finite subset $\pi$ of $\mathcal{B}$ is called a $\mathcal{B}$-partition if for distinct pairs $\left(I^{\prime}, x^{\prime}\right)$ and $\left(I^{\prime \prime}, x^{\prime \prime}\right)$ in $\pi$, the intervals $I^{\prime}$ and $I^{\prime \prime}$ are nonoverlapping. If $\cup_{(I, x) \in \pi} I=\Delta, \pi$ is called a $\mathcal{B}$-partition of $\Delta$.

Given an interval function $F$ defined on the family of all $\alpha$-regular intervals, a set $E \subset \Delta$ and a gauge $\delta$, for any interval $A \in \mathcal{I}$ we put

$$
V_{\delta}(A)=V\left(\mathcal{F}_{\delta}^{\alpha}, F, E, A\right)=\sup \left\{\sum_{(I, x) \in \pi}|F(I)|: \pi \subset \mathcal{F}_{\delta}^{\alpha}[E] \cap \mathcal{F}_{\delta}^{\alpha}(A)\right\}
$$

and we call it the $\delta$-variation over $\mathcal{F}^{\alpha}$ of the function $F$ on $E \cap A$. If $E \cap A=\emptyset$, we define $V_{\delta}(A)=0$. Clearly, for each fixed $E$, the interval function $V_{\delta}(A)$ is non-negative and superadditive.

Definition 1. An interval function $F$ defined on the family of all $\mathcal{B}$-intervals is said to be $\mathcal{B}$-continuous at a point $x$ if for any $\varepsilon>0$ there exists $\eta>0$ such that $|F(I)|<\varepsilon$ whenever $(I, x) \in \mathcal{B}_{\eta}[\{x\}]$.

We need the following particular case of a result established in [1].

Proposition 1. Let $F$ be a $\mathcal{F}^{\alpha}$-continuous additive function defined on the family of all $\alpha$-regular intervals. Then for a fixed set $E \subset \Delta$ and a fixed gauge $\delta$ the interval function $V_{\delta}(I)=V\left(\mathcal{F}_{\delta}^{\alpha}, F, E, I\right)$ is $\mathcal{F}$-continuous at each point $x \in \Delta$.

\section{Main Results}

To define the "ordinary" Perron integral we recall first the definitions of $\alpha$ regular and ordinary lower and upper derivates.

The $\alpha$-regular lower and upper derivates of an interval function $F$ at a point $x$ (i.e., the derivates with respect to the basis $\mathcal{F}^{\alpha}$ ) are defined respectively as

$$
\begin{aligned}
& \underline{D}_{\alpha} F(x)=\sup _{\delta} \inf \left\{\frac{F(I)}{|I|}:(I, x) \in \mathcal{F}_{\delta}^{\alpha}[\{x\}]\right\}, \\
& \bar{D}_{\alpha} F(x)=\inf _{\delta} \sup \left\{\frac{F(I)}{|I|}:(I, x) \in \mathcal{F}_{\delta}^{\alpha}[\{x\}]\right\} .
\end{aligned}
$$

If $\underline{D}_{\alpha} F(x)=\bar{D}_{\alpha} F(x) \neq \pm \infty$, we call this common value the $\alpha$-regular derivative $D_{\alpha} F(x)$ of $F$ at $x$ and we say that $F$ is $\alpha$-regularly differentiable at $x$. 
The ordinary lower and upper derivates are defined respectively as

$$
\begin{aligned}
& \underline{D} F(x)=\inf _{\alpha \in(0,1)} \underline{D}_{\alpha} F(x), \\
& \bar{D} F(x)=\sup _{\alpha \in(0,1)} \bar{D}_{\alpha} F(x) .
\end{aligned}
$$

If $\underline{D} F(x)=\bar{D} F(x) \neq \pm \infty$, we call this common value the ordinary derivative $D F(x)$ of $F$ at $x$ and we say that $F$ is ordinarily differentiable at $x$. (Note that this definition is obviously equivalent to the one given in [16].)

It is clear that for any $0<\alpha<\beta<1$ and for any $x \in \Delta$ we have

$$
\underline{D} F(x) \leq \underline{D}_{\alpha} F(x) \leq \underline{D}_{\beta} F(x) \leq \bar{D}_{\beta} F(x) \leq \bar{D}_{\alpha} F(x) \leq \bar{D} F(x) .
$$

Definition 2. Let $f$ be a point function on $\Delta$. An interval function $M$ (resp. $m$ ) is called a major (resp. a minor) function of $f$ on $\Delta$ if it is superadditive (resp. subadditive) and the lower (resp. upper) ordinary derivate satisfies the inequality

$$
\underline{D} M(x) \geq f(x) \quad(\text { resp. } \bar{D} m(x) \leq f(x)) \text { for all } x \in \Delta .
$$

A function $f$ is said to be P-integrable on $\Delta$ if

$$
-\infty<\inf _{M}\{M(\Delta)\}=\sup _{m}\{m(\Delta)\}<\infty,
$$

where "inf" is taken over all major functions $M$ and "sup" is taken over all minor functions $m$. The common value is denoted by $(P) \int_{\Delta} f$, and is called the ordinary Perron integral or P-integral of $f$ over $\Delta$.

Since $f$ is also integrable on each subinterval of $\Delta$, for each $I \in \mathcal{I}$ we can define the indefinite $P$-integral $F(I)=(P) \int_{I} f$ which is an additive function on $\mathcal{I}$.

The consistency of Definition 2 follows from the fact that for any major function $M$ and any minor function $m$ the difference $R=M-m$ is a superadditive interval function for which $\underline{D} R(x) \geq 0$ at each point $x \in \Delta$, and from the following assertion.

Proposition 2. If a superadditive interval function $R$ satisfies the inequality $\underline{D} R(x) \geq 0$ at each point $x$ of an interval $I$, then $R(I) \geq 0$.

The proof is the same as the one of the similar assertion in [16, p. 190], for an additive interval function. 
In the previous definition replacing lower and upper ordinary derivates with $\alpha$-regular lower and upper derivates for a fixed regularity $\alpha \in(0,1)$, we get the known definition of $\alpha$-regular Perron integral ( $P_{\alpha}$-integral) (see [13]).

It is clear from (3) that any major function in the sense of Definition 2 also is a $P_{\alpha}$-major function and the same is true for minor functions. Hence each $P$-integrable function is $P_{\alpha}$-integrable, $\alpha \in(0,1)$, with the same integral. We denote this fact symbolically by the inclusion

$$
P \subseteq \bigcap_{\alpha \in(01)} P_{\alpha}
$$

To define the "continuous" version of $P$-integral we introduce an appropriate version of continuity of an interval function at a point which we call regular continuity.

Definition 3. We say that an interval function is regularly continuous at a point $x$ if it is $\mathcal{F}^{\alpha}$-continuous at this point for each $\alpha \in(0,1)$.

Now we get the definition of $P^{c}$-integral if we replace major and minor functions in the Definition 2 with regularly continuous major and minor functions. Obviously any $P^{c}$-integrable function is $P$-integrable and we can write

$$
P^{c} \subseteq P .
$$

In a similar way we get the definition of $P_{\alpha}^{c}$-integral using $\mathcal{F}^{\alpha}$-continuous major and minor functions (see Definition 1).

As we have already mentioned in the Introduction, it is proved in [12] that the $P_{\alpha}$-integral is equivalent to the $P_{\alpha}^{c}$-integral, and our aim here is to prove the equivalence of the $P$-integral and the $P^{c}$-integral. Our method is based on the Kurzweil-Henstock approach to the notion of integral and we need some definitions and known facts from this theory.

The $P_{\alpha}$-integral is known (see for example [13]) to be equivalent to the following version of the $n$-dimensional Kurzweil-Henstock integral.

Definition 4. Let $\alpha \in(0,1)$ be fixed. A function $f$ on $\Delta$ is called $H_{\alpha^{-}}$ integrable on $\Delta$ with integral $A$ if we can find a gauge $\delta$ so that

$$
\left|\sum_{(x, I) \in \pi} f(x)\right| I|-A|<\varepsilon
$$

for any $\mathcal{F}_{\delta}^{\alpha}$-partition $\pi$ of $\Delta$. We put $A=\left(H_{\alpha}\right) \int_{\Delta} f$. 
The following integral was introduced by Mawhin in [11] where it was named $G P$-integral. In [9] it is called the $M$-integral, and we prefer to use the same name for this integral.

Definition 5. A function $f$ on $\Delta$ is called $M$-integrable on $\Delta$ with integral $A$ if for each $\alpha \in(0,1) f$ is $H_{\alpha}$-integrable on $\Delta$ with integral $A$. We put $A=(M) \int_{\Delta} f$.

The following proposition is an immediate consequence of the previous definition and the Saks-Henstock lemma for the $H_{\alpha}$-integral (see [8]).

Proposition 3. If a function $f$ is $M$-integrable on $\Delta$ and $F$ is its indefinite $M$-integral, then for any $\varepsilon>0$ and for any $\alpha \in(0,1)$ there exists a gauge $\delta$ such that for any partition $\pi \subset \mathcal{F}_{\delta}^{\alpha}$ we have

$$
\sum_{(x, J) \in \pi}|f(x)| J|-F(J)|<\varepsilon .
$$

It is easy to proof that $M$-integrability of a function and the value of the $M$-integral does not depend on the values of the function on a set of measure zero. So having a fixed set $E$ of measure zero, we can assume that an integrable function is equal to zero on $E$. This observation together with Proposition 3 gives us the next result.

Proposition 4. Let $F$ be the indefinite $M$-integral of some integrable function on $\Delta$ and let $E \subseteq \Delta$ be a set of measure zero. Then for any $\varepsilon>0$ and for any $\alpha \in(0,1)$ there exists a gauge $\delta$ such that $V\left(\mathcal{F}_{\delta}^{\alpha}, F, E, \Delta\right)<\varepsilon$.

From Definition 5 and from the equivalence of the $H_{\alpha}$-integral and the $P_{\alpha}$-integral we get $M=\bigcap_{\alpha \in(01)} P_{\alpha}$. This equality together with (4) gives

$$
P \subseteq \bigcap_{\alpha \in(01)} P_{\alpha}=M
$$

So we have proved the following proposition.

Proposition 5. Any P-integrable function on $\Delta$ is also $M$-integrable on $\Delta$, and the two integrals coincide.

Our next step is to prove the following.

Proposition 6. Any $M$-integrable function on $\Delta$ is also $P^{c}$-integrable on $\Delta$, and the two integrals coincide. 
Proof. Let $f$ be an $M$-integrable function on $\Delta$ and $F$ its indefinite integral. As $f$ is also $H_{\alpha}$-integrable, for any $\alpha \in(0,1)$, it follows that $F$ is $\alpha$-regularly differentiable and $D_{\alpha} F(x)=f(x)$ almost everywhere on $\Delta$ (see [13]). Moreover, as it is proved in [10], the $\alpha$-regular differentiability of any additive interval function defined on $\mathcal{I}$ does not depend on $\alpha$ in the interior of $\Delta$; i.e., if $F$ is $\alpha$-regularly differentiable at $x$, then it is $\beta$-regularly differentiable at $x$ to the same value, for any $\beta \in(0,1)$. Hence $F$ is ordinarily differentiable to $f$ almost everywhere on $\Delta$.

Fix $\varepsilon>0$ and denote by $E$ the set on which $D F$ does not exist or is not equal to $f$. Then $|E|=0$. Take $\alpha_{n} \searrow 0$ with $\alpha_{n} \in(0,1)$. Now to $F$, to each $\alpha_{n}$ and to the chosen $E$ we apply Proposition 4 with $\varepsilon$ replaced by $\varepsilon / 2^{n+1}$. In this way we get a sequence of gauges $\delta_{n}$ such that for the corresponding sequence of interval functions $V_{\delta_{n}}(I)=V\left(\mathcal{F}_{\delta_{n}}^{\alpha_{n}}, F, E, I\right)$ we have

$$
V_{\delta_{n}}(\Delta) \leq \frac{\varepsilon}{2^{n+1}} .
$$

Define on $\mathcal{I}$ the function $R=\sum_{n=1}^{\infty} V_{\delta_{n}}$. According to (7) we have

$$
0 \leq R(\Delta) \leq \frac{\varepsilon}{2} \text {. }
$$

As each $V_{\delta_{n}}$ is obviously superadditive, so is $R$. Moreover $R$ is $\mathcal{F}$-continuous at each point of $\Delta$. In fact, for each $n$, we can apply Proposition 1 to $\mathcal{F}^{\alpha_{n}}$ and to the gauge $\delta_{n}$ to deduce that $V_{\delta_{n}}$ is $\mathcal{F}$-continuous. This easily implies the $\mathcal{F}$-continuity of $R$.

We can also construct in a standard way a nonnegative additive $\mathcal{F}$-continuous interval function $G$ defined on $\mathcal{I}$ such that $\underline{D} G(x)=+\infty$ for each $x \in E$ and

$$
G(\Delta)<\frac{\varepsilon}{2} .
$$

Now we prove that the function $M=F+R+G$ is a superadditive regularly continuous major function of $f$. The superadditivity of $M$ follows from the superadditivity of $R$ and the additivity of $F$ and $G$. Recall that $F$ is the indefinite $H_{\alpha}$-integral of $f$, for each $\alpha \in(0,1)$. Hence it is $\mathcal{F}^{\alpha}$-continuous (see [13]), for each $\alpha \in(0,1)$ and therefore, by Definition 3, is also regularly continuous. The regular continuity of $R$ and $G$ follows from their $\mathcal{F}$-continuity. This implies the regular continuity of $M$.

We prove next that

$$
\underline{D} M(x) \geq f(x)
$$

for all $x \in \Delta$.

If $x \in \Delta \backslash E$ then (10) holds since at such a point $D F(x)=f(x), \underline{D} R(x) \geq 0$ and $\underline{D} G(x) \geq 0$. If $x \in E$, then having chosen $\alpha \in(0,1)$, we fix $n$ for which 
$\alpha_{n} \leq \alpha$ and an interval $I$ such that $(I, x) \in \mathcal{F}_{\delta_{n}}^{\alpha_{n}}[\{x\}]$. For such an interval we obviously have $|F(I)| \leq V_{\delta_{n}}(I)$. Then $F(I)+V_{\delta_{n}}(I) \geq 0$. Hence

$$
\frac{M(I)}{|I|}=\frac{F(I)+R(I)+G(I)}{|I|} \geq \frac{F(I)+V_{\delta_{n}}(I)+G(I)}{|I|} \geq \frac{G(I)}{|I|} .
$$

From this, from (3) and from the properties of $G$ we infer

$$
\underline{D}_{\alpha} M(x) \geq \underline{D}_{\alpha_{n}} M(x) \geq \underline{D}_{\alpha_{n}} G(x) \geq \underline{D} G(x)=+\infty>f(x) .
$$

As $\alpha$ is arbitrary, the last inequality implies (10) for $x \in E$. So (10) holds for every $x \in \Delta$.

Therefore we have proved that $M$ is a regularly continuous major function of $f$. Moreover (8) and (9) imply

$$
0 \leq M(\Delta)-F(\Delta)<\varepsilon
$$

In a similar way we can show that the subadditive function $m=F-R-G$ is a regularly continuous minor function of $f$ with $0 \leq F(\Delta)-m(\Delta)<\varepsilon$. From the last inequality together with (11) we get that $f$ is $P^{c}$-integrable and $F$ is its $P^{c}$-integral.

The last proposition together with (5) and (6) gives us the following chain of inclusions. $P \subseteq M \subseteq P^{c} \subseteq P$. So we have proved the main result of this paper.

Theorem 1. The P-integral is equivalent to the $P^{c}$-integral, and both are equivalent to the Mawhin integral.

\section{Some Additional Remarks}

1. Although the indefinite $M$-integral of a function $M$-integrable on $\Delta$ is additive on $\mathcal{I}$, this integral is not additive in a stronger; namely, if $J$ and $K$ are nonoverlapping intervals such that $J \cup K$ is an interval, then a function $f: J \cup K \rightarrow \mathbb{R}$ needs not be $M$-integrable on $J \cup K$ even if both its restrictions $\left.f\right|_{J}$ and $\left.f\right|_{K}$ are $M$-integrable on $J$ and $K$ respectively.

In [9] J. Kurzweil and J. Jarník have suggested a simple way to remove this drawback by introducing the so-called extensive $M$-integral.

Definition 6. A function $f: \Delta \rightarrow \mathbb{R}$ is said to be extensively $M$-integrable on $\Delta$ if there exists a compact interval $L \subseteq \mathbb{R}^{n}$ such that $\Delta \subseteq \operatorname{Int} L$ and the function

$$
f_{\text {ex }}(x)= \begin{cases}f(x) & \text { for } x \in \Delta, \\ 0 & \text { for } x \in L \backslash \Delta\end{cases}
$$

is $M$-integrable on $L$. 
In the same way we can define the extensive P-integral and the extensive $P^{c}$-integral.

In [9] it is proved that the extensive $M$-integral is equivalent to the Pfeffer integral defined in [15] (see also [14]). Thus we can apply the technics of the previous section to construct continuous major and minor functions for the extensive $P$-integral, the extensive Mawhin integral and consequently for the Pfeffer integral of [15].

2. Using the equivalence of the $P$-integral to the $M$-integral we can give a descriptive characterization of the $P$-integral in terms of the absolute continuity of the variational measure generated by the indefinite integral $F$. We recall that by the variational measure we mean the set function

$$
V_{F}(E)=\sup _{\alpha} \inf _{\delta} V\left(\mathcal{F}_{\delta}^{\alpha}, F, E, \Delta\right) .
$$

In $[4$, Theorem 6], it is proved that an additive function $F$ is the indefinite $M$ integral of its ordinary derivative iff the variational measure $V_{F}$ is absolutely continuous with respect to Lebesgue measure. So using our Theorem 1 we get the following.

Theorem 2. A function $f: \Delta \rightarrow \mathbb{R}$ is P-integrable iff there exists an additive interval function $F$ on $\mathcal{I}$ (its indefinite integral) which generates a variational measure absolutely continuous with respect to Lebesgue measure and for which $D F(x)=f(x)$ almost everywhere in $\Delta$.

Note that in the "if" part of this theorem we need not require the function $F$ to be differentiable. The differentiability of $F$ is here a consequence of the absolute continuity of the variational measure.

3. We remark in conclusion that the question of whether the Perron integral, defined with respect to a general basis, is equivalent or not to the one defined by continuous (with respect to this basis) major and minor functions, is still open (see [3], p.202 and [6]).

\section{References}

[1] B. Bongiorno, L. Di Piazza and V. Skvortsov, On continuous major and minor functions for the n-dimensional Perron integral, Real Anal. Exch., 22 (1) (1996/97), 318-327.

[2] B. Bongiorno, L. Di Piazza and V. Skvortsov, On variational measures related to some bases, J. Math. Anal. Appl., 250 (2000), 533-547. 
[3] P. S. Bullen et al.(ed), New integrals, Lecture Notes in Math., 1419, Springer-Verlag, 1990.

[4] L. Di Piazza, Variational measures in the theory of integration in $\mathbb{R}^{m}$, Czechoslovak Math. J., to appear.

[5] C. A. Faure and J. Mawhin, The Hake's property for some integrals over multidimensional intervals, Real Anal. Exch., 20(2) (1994-95), 622-630.

[6] Z. T. Gong, On a problem of Skvortsov involving the Perron Integral, Real Anal. Exch., 17(2) (1991-92), 748-750.

[7] J. Jarník, J. Kurzweil and S. Schwabik, On Mawhin's approach to multiple nonabsolutely convergent integral, Casopis Pest. Mat., 108 (1983), 356380 .

[8] J. Kurzweil and J. Jarník, Equiintegrability and controlled convergence of Perron-type integrable functions, Real Anal. Exch., 17 (1991-92), 110139.

[9] J. Kurzweil and J. Jarník, Equivalent definitions of regular generalized Perron integral, Czechoslovak Math. J., 42 (1992), 365-378.

[10] J. Kurzweil and J. Jarník, Differentiability and integrability in n dimensions with respect to $\alpha$-regular intervals, Results Math., 21 (1-2) (1992), $138-151$.

[11] J. Mawhin, Generalized multiple Perron integrals and the Green-Goursat theorem for differentiable vector fields, Czechoslovak Math. J., 31(106) (1981), 614-632.

[12] M. P. Navarro and V. A. Skvortsov, On n-dimensional Perron integral, Southeast Asian Math. Bull., 20 (2) (1997), 111-116.

[13] K. M. Ostaszewski, Henstock integration in the plane, Memoirs Amer. Math. Soc., Providence, 353, 1986.

[14] W. F. Pfeffer, The divergence theorem, Trans. AMS, 295 (1986), 665-685.

[15] W. F. Pfeffer, A Riemann type integration and the fundamental theorem of calculus, Rendiconti Circolo Mat. Palermo, Serie II 36 (1987), 482-506.

[16] S. Saks, Theory of the integral, Dover, New York, 1964.

[17] V. A. Skvortsov, Continuity of $\delta$-variation and construction of continuous major and minor functions for the Perron integral, Real Anal. Exch., 21(1), (1995/96), 270-277. 